\title{
Gabriele Diewald
}

\section{Konstruktionen und Paradigmen ${ }^{1}$}

\author{
Abstract \\ The notion of constructions - as the basic semiotic units of language varying in complexity, \\ size and degree of abstraction-has proved to be useful in diachronic research in general and \\ in grammaticalization studies in particular. Grammaticalization scenarios have been mod- \\ elled as a unidirectional succession of specific types of constructions, thus providing a \\ means for depicting the interdependence of pragmatic and contextual feature on the one \\ hand and language internal features on the other. However, the endpoint of grammaticali- \\ zation processes, i.e. the integration of formerly autonomous signs into tightly organized \\ paradigmatic structures (grammatical paradigms), so far has not been in the scope of con- \\ structional approaches. It is argued that existing models of grammaticalization scenarios \\ should be expanded by a fourth stage, the stage of paradigmatic integration. It is suggested \\ that a grammatical paradigm represents a particular type of construction. Thus, the stage of \\ paradigmatic integration represents the formation of a fourth type of construction. \\ 0. Einleitung, Grundlagen, Thesen \\ 1. Konstruktionen \\ 2. Kontexte und Konstruktionen bei grammatikalisierendem Sprachwandel \\ 3. Paradigmatisierung als notwendige vierte Stufe in Grammatikalisierungsprozessen \\ 4. Das grammatische Paradigma als Konstruktion \\ 5. Literatur
}

\section{Einleitung, Grundlagen, Thesen}

Dieser Beitrag verbindet zwei zentrale, jedoch gleichermaßen schwierige Begriffe der jüngeren Grammatikalisierungsforschung: Konstruktionen und Paradigmen. Sie sollen im Folgenden für diachrone Fragestellungen der Grammatikalisierungsforschung, insbesondere zur Erfassung der Zielkategorien von Grammatikalisierungsvorgängen, in einen systematischen Zusammenhang gebracht werden. Anknüpfend an frühere Arbeiten zur Abfolge von Konstruktions- bzw. Kontexttypen in Grammatikalisierungsprozessen wird exploriert, inwiefern auch der Zielpunkt von Grammatikalisierungsvorgängen unter konstruktionsgrammatischer Perspektive zu fassen ist. Bevor dies geschieht, ist es sinnvoll, einige Grundannahmen und Definitionen, die Ausgangspunkt dieser Untersuchung bilden, kurz zu erläutern.

Grammatikalisierung ist definiert als Sprachwandelprozess in Richtung auf eine stärkere grammatische Funktionalität eines Zeichens bzw. einer Konstrukti-

1 Diese Studie wurde unterstützt im Rahmen der Belgian Science Policy (Interuniversity Attraction Poles, project GRAMIS P6/44). 
on, wobei zugleich lexikalische Funktion(en) abgebaut werden. Grammatikalisierung bedeutet somit das „Grammatik-Werden linguistischer Einheiten“. Dabei wird, ungeachtet der Gradienz zwischen lexikalischen und grammatischen Zeichen, eine prinzipielle Trennbarkeit beider Klassen von Zeichen in formaler und funktionaler Hinsicht vorausgesetzt. ${ }^{2}$

Die entscheidenden Phasen von Grammatikalisierungsprozessen können gewinnbringend unter Zuhilfenahme des Konstruktionsbegriffs dargestellt werden. Erste Modelle hierzu wurden in Diewald 2006a, 2006b, Traugott 2008, ${ }^{3}$ und Traugott [demn.] vorgeschlagen. Ein wesentlicher methodologischer Vorzug der Anwendung des Konstruktionsbegriffs für Grammatikalisierungsfragen liegt in seiner flexiblen Granularität, die es ermöglicht, die Grenzen zwischen linguistischen Einheiten unterschiedlicher Strukturebenen bzw. unterschiedlicher Wertigkeiten aufzuheben, so z.B. zwischen Wortformen und Syntagmen oder zwischen Idiomen und produktiven Strukturen.

In der bislang geführten Diskussion zu Konstruktionen in Grammatikalisierungsvorgängen, werden Konstruktionen vor allem in ihrer syntagmatischen Dimension wahrgenommen, d.h. als der relativ flexible linguistisch-pragmatische Kontext, in dem sich grammatikalisierungsrelevanter Wandel vollzieht. Soll jedoch auch die Zielkategorie von Grammatikalisierungsprozessen erfasst werden, dann ist - so die hier vertretene These - die Berücksichtigung der paradigmatischen Achse notwendig.

Denn grammatische Kategorien, also die Zielpunkte von Grammatikalisierungsprozessen, sind - ungeachtet der Problematik dieser Begriffe (s.u.) - maßgeblich über die Kriterien der Obligatorik und Paradigmatizität definiert, die allgemein als hochrelevante Indikatoren für den Grad der Grammatikalität von linguistischen Einheiten betrachtet werden. Generell gilt daher folgende Aussage: „Grammatische Bedeutung ist in geschlossenen Paradigmen organisiert, die obligatorisch zu realisieren sind“". ${ }^{4}$

2 Zur Illustration seien einige einschlägige Zitate, die sich beliebig vermehren ließen, angeführt: „Grammaticalization is a process leading from lexemes to grammatical formatives. A number of semantic, syntactic and phonological processes interact in the grammaticalization of morphemes and of whole constructions.“ (Lehmann 1995[1982]: V) „Grammaticalization is the process by which constructions with specific lexical items develop grammatical functions, leading to the reinterpretation of the lexical items as possessing grammatical functions.“ (Croft 2000: 156) „To conclude, there is evidence to suggest that grammaticalization can be defined as a distinct process, leading to the rise and development of new grammatical forms.“ (Heine 2003: 284)

3 Traugott (2008b: 7) hebt hervor, dass Grammatik in konstruktionsgrammatischer Interpretation insgesamt konstruktionsförmig ist und ein „holistisches Gebilde“ darstellt, bei dem „Morphosyntax, Phonologie, Semantik und auch (in den meisten Versionen der Konstruktionsgrammatik) Pragmatik auf der Ebene der Konstruktion miteinander verklammert [sind]“.

4 Von den vielen Autoren, die hier angeführt werden könnten, seien beispielshalber genannt: Bybee/Perkins/Pagliuca (1994: 2), Haspelmath (1998: 318), Dahl (2000, 2001), Plungian (1998), Mel'čuk (1976: 84), Radtke (1998: 10). 
Die genannten Kriterien nehmen auch in Lehmanns Grammatikalisierungskonzeption unter dem Stichwort der Paradigmatizität bzw. des Grammatikalisierungsparameters der paradigmatischen Variabilität einen zentralen Platz ein, ${ }^{5}$ wie folgendes Zitat illustriert:

The cohesion of a sign with other signs in a paradigm will be called its paradigmaticity, that is, the degree to which it enters a paradigm, is integrated into it and dependent on it. [...] The paradigmatic variability of a sign is the possibility of using other signs in its stead or of omitting it altogether. (Lehmann 2002a: 110)

Paradigmatizität ist, wie später noch ausgeführt wird, die wesentliche Eigenschaft, die grammatische von lexikalischen Zeichen unterscheidet, da letztere zwar in lose organisierten, offenen Wortfeldern, nicht aber in Paradigmen organisiert sind. Die Zunahme an Paradigmatizität (die von einer Einheit immer nur mit Bezug auf ihre paradigmatischen Partner festgestellt werden kann) ist somit ein wesentliches Merkmal stärkerer Grammatikalisierung.

Paradigmatizität ist wie Obligatorik ein graduelles Phänomen, das nicht immer leicht zu ermitteln ist. Daher hat es zu Recht immer auch Warnungen davor gegeben, den Faktor der paradigmatischen Einbindung und Obligatorik unhinterfragt als zentrales bzw. einziges Definiens zu verwenden. Insbesondere Wiemer/Bisang (2004: 5) und Himmelmann (1992: 18), (2004: 33) relativieren die Zentralität der Begriffe Paradigma und Obligatorik. Andere Autoren, wie Lehmann (1982: 12) und Plungian 1998 halten fest, dass beide Begriffe - Obligatorik und Paradigma - auf Phänomene bezogen sind, die in sich gradueller Natur sind. Grammatische Kategorien können somit mehr oder weniger strenge Paradigmen bilden, und sie können mit einem größeren oder kleineren Ausmaß an Obligatorik verbunden sein (vgl. auch Lehmann (1995 [1982]: 12).

An einem Ende des Spektrums, am Pol maximaler Obligatorik, befinden sich Paradigmen, deren Auswahl vollständig automatisierten grammatischen Regeln, die häufig unmotiviert scheinen, unterworfen ist. Klassische Beispiele hierfür sind die morphologischen Paradigmen der Kasusmarkierung von Nomina bzw. Nominalphrasen im Deutschen, oder die Genuszuordnung bei Nomina und die entsprechende Genuskongruenz von Adjektiven im Deutschen. Am anderen Pol stehen Kategorien wie das Passiv im Deutschen, das niemals obligatorisch im Sinne der zuvor genannten Beispiele ist. Ungeachtet dieser Relativierungen bieten beide Begriffe eine erste, hinreichend tragfähige Grundlage für eine schematische Definition der Zielkategorien von Grammatikalisierungsprozessen (vgl. ausführlich Diewald 2008a, [im Druck]).

Im Folgenden soll auf der Basis dieser Vorüberlegungen zum einen der Konstruktionsbegriff geschärft werden, in dem die paradigmatische Komponente

5 Im System der Grammatikalisierungsparameter bestimmt Lehmann Paradigmatizität und Obligatorik über den Verlust der Autonomie eines Zeichens: „Grammaticalization of a linguistic sign is a process in which it loses in autonomy by becoming subject to constraints of the linguistic system.“ (Lehmann 2004: 155) 
stärker berücksichtigt wird. Zum anderen soll das bisher erarbeitete dreistufige Modell eines über Kontext-/Konstruktionstypen definierten Grammatikalisierungsszenarios weiterentwickelt werden, um die letzte, bislang vernachlässigte Stufe von Grammatikalisierungsprozessen, nämlich die der Neuformation bzw. Neuordnung eines Paradigmas durch die hinzukommende Einheit, zu integrieren. Kurz, beide Konzepte, Paradigmen und Konstruktionen, werden in einen weiterführenden Zusammenhang gebracht, um zur Lösung eines wichtigen Problems der Grammatikalisierung beizutragen, nämlich eine Verbindung zu schaffen zwischen den prozesshaften Vorgängen und prozessorientiert formulierten theoretischen Konzepten der Grammatikalisierungsforschung und dem statischstrukturellen Konzept des Paradigmas bzw. der Grammatik als „fertiges“, stabiles System.

Die zentralen Thesen dieses Beitrags sind:

1. Das bisher entwickelte dreistufige Grammatikalisierungsszenario benötigt eine vierte Stufe, um Grammatikalisierungsprozesse eindeutig von anderen Sprachwandelvorgängen zu unterscheiden.

2. Diese vierte Stufe betrifft die Integration in ein grammatisches Paradigma, also den Prozess der Paradigmatisierung, wobei die paradigmatische Einordnung als zentrale (wenn auch graduelle) Eigenschaft grammatischer Elemente definiert wird.

3. Paradigmen sind Konstruktionen im konstruktionsgrammatischen Sinn. Auf der Skala der Abstraktheit bzw. Schematizität befinden sie sich am oberen (schematischen und abstrakten) Ende.

Die Gliederung des Beitrags folgt im Wesentlichen den oben genannten Punkten. Nach einem kurzen Blick auf konstruktionsgrammatische Konzepte und Forschungsergebnisse (1), wird das Kontextmodell der Grammatikalisierungsstufen kurz rekapituliert (2) und seine Erweiterung um eine vierte Stufe erläutert (3). Schließlich wird dargelegt, inwiefern grammatische Paradigmen einen bestimmten Typus von Konstruktionen darstellen (4).

\section{Konstruktionen}

Das hier vorgestellte Modell baut auf früheren Überlegungen zu Konstruktionen und Kontexten in Grammatikalisierungsprozessen auf (s. Lehmann 1985, 1992 406, Bybee, Perkins \& Pagliuca 1994: 11, Bisang 1998: 20, Traugott 2003, Traugott 2008a, Diewald 2002, 2006a, 2006b, 2008b). Der verwendete Konstruktionsbegriff orientiert sich an der weithin als geeignete Grundlage erachteten Position von Goldberg, die durch folgende Zitate skizziert sei und die in ihren hier interessierenden Aspekten durch die Diskussionen der letzten Jahre hindurch konstant geblieben ist: 
According to Construction Grammar, a distinct construction is defined to exist if one or more of its properties are not strictly predictable from knowledge of other constructions existing in the grammar: $\mathrm{C}$ is a construction iff $\mathrm{def}_{\mathrm{C}} \mathrm{C}$ is a form-meaning pair $<\mathrm{F}_{\mathrm{i}}, \mathrm{S}_{\mathrm{i}}>$ such that some aspect of $\mathrm{F}_{\mathrm{i}}$ or some aspect of $\mathrm{S}_{\mathrm{i}}$ is not strictly predictable from C's component parts or from other previously established constructions. (Goldberg 1995: 4)

Any linguistic pattern is recognized as a construction as long as some aspect of its form or function is not strictly predictable from its component parts or from other constructions recognized to exist. In addition, patterns are stored as constructions even if they are fully predictable as long as they occur with sufficient frequency. (Goldberg 2006: 5)

Diese Definition stimmt in ihren Grundannahmen mit den meisten konstruktionsgrammatischen Auffassungen überein, z.B. Kay/Fillmore (1999: 2f.), Croft (2001: 18f.; 261), Michaelis (2004: 8), Fried/Östman $2004 .^{6}$

Wie unschwer zu erkennen ist, ist dieser Konstruktionsbegriff stark syntagmatisch ausgerichtet. Ein entscheidender Punkt von Beginn der Diskussion an war es, das Kontinuum zwischen Lexik und Grammatik abzubilden, was insbesondere in folgendem Zitat explizit gemacht wird:

To adopt a constructional approach is to undertake a commitment in principle to account fort he entirety of each language. This means that the relatively general patterns of language, such as the one licensing the ordering of a finite auxiliary verb before its subject in English as illustrated in 1 and the more idiomatic patterns, such as those exemplified in 2, stand on an equal footing as data for which the grammar must provide an account. (Kay \& Fillmore 1999: 1)

Insgesamt wird die Summe der Konstruktionen in einer Sprache als ein Kontinuum von generellen, abstrakten und transparenten Konstruktionen an einem Ende der Skala bis zu spezifischen, idiosynkratischen und nicht-durchsichtigen Konstruktionen konzipiert (vgl. auch Traugott 2008a: 7). Diesem Kontinuum werden zur Orientierung und Operationalisierung bestimmte prototypische Stufen bzw. Abstraktionsgrade zugewiesen, die jedoch in der Literatur unterschied-

6 Die Modifikationen in Goldberg (2006) gegenüber dem Modell von 1995 betreffen u.a. die Gewichtung der Bedingung der Nicht-Kompositionalität für den Konstruktionsbegriff. Goldberg (2006) nimmt hier eine weniger strenge Position als in früheren Arbeiten ein und hält die NichtKompositionalität nicht mehr für ein notwendiges Definiens von Konstruktionen. Dies wird aus folgendem Zitat deutlich (unter dem Konzept der unpredictability: „Thus evidence that a word or pattern is not strictly predictable provides sufficient evidence that the form must be listed as a construction in what is sometimes called the „constructicon“ $[\ldots]$. At the same time, unpredictability is not a necessary condition for positing a stored construction. There is evidence from psycholinguistic processing that patterns are also stored if they are sufficiently frequent, even when they are fully regular instances of other constructions and thus predictable [... “. . Da im hier vertretenen Ansatz - im Einklang mit den Grundsätzen der Grammatikalisierungsforschung schon immer von der prinzipiellen Gradualität aller relevanten Merkmale ausgegangen wurde, ist diese Modifikation unproblematisch. Sie hat inzwischen breite Resonanz in der Forschung gefunden (vgl. Croft 2005: 274, Trousdale 2008a: 42f.). 
lich bestimmt werden. Da diese Diskussion für das Konzept des Paradigmas nicht unerheblich ist, sei sie kurz skizziert.

Kay und Fillmore (1999: 2f.) unterscheiden in ihren frühen und äußerst einflussreichen Arbeiten zunächst zwischen „constructs“ und „constructions“ und treffen damit eine Unterscheidung, die, wie das folgende Zitat zeigt, der Trennung zwischen token und type nahe kommt: „A construction (e.g. the subjectauxiliary inversion construction) is a set of conditions licensing a class of actual constructs of a language (e.g. the class of English inverted clauses...)." Dabei sind construct „the words, phrases and sentences of the language which they [i.e. the constructions] license" und zugleich „utterance-types of the language" und constructions „rooted trees whose nodes correspond to feature structures“, also „pieces of grammar“ (Kay \& Fillmore 1999: 2f.).

Für diachrone Untersuchungen hat sich weiterhin die Einteilung von Konstruktionstypen nach Idiomatizitätsgraden als sehr nützlich erwiesen, wie sie in der Studie von Fillmore, Kay und O'Connor (1988) vorgeschlagen wird. Die Autoren unterscheiden zwischen Konstruktionstypen mit verschiedenen Graden der Idiomatizität bzw. Idiosynkrasie, wobei ein absolut „,reines“, d.h. in keiner Weise kompositionelles Idiom eine völlige Übereinstimmung der tokens mit dem type aufweisen würde.

Als eine Untergruppe, die für Grammatikalisierungsfragen äußerst wichtig ist, nennen die Autoren „formal or lexically open idioms“, und definieren sie als „syntactic patterns dedicated to semantic and pragmatic purposes not knowable from their form alone“ (Fillmore, Kay \& O’Connor 1988: 505). Formale idiomatische Konstruktionen sind somit Konstruktionen, deren Kompositionalität reduziert ist, d.h. die zumindest teilweise Form-Bedeutungszuordnungen zeigen, die holistisch zu interpretieren sind und nicht gänzlich aus der Kombination ihrer Bestandteile (d.h. anderer Konstruktionen) abgeleitet werden können. Dennoch sind formale idiomatische Konstruktionen voll produktiv, da ihre syntaktischen Positionen nicht mit bestimmten lexikalischen Einheiten belegt sind.

Ein zweiter Typ von Konstruktionen, die im hier verfolgten Kontext wichtig sind, sind die sogenannten ,extragrammatical idioms“, die die Autoren folgendermaßen definieren:

Such expressions [i.e. extragrammatical idioms] have grammatical structure, to be sure, but the structures they have are not made intelligible by knowledge of the familiar rules of the grammar and how those rules are most generally applied (Fillmore, Kay \& O’Connor 1988: 505).

Beide Typen idiomatischer Konstruktionen sind an Grammatikalisierungsvorgängen beteiligt und korrelieren, wie in früherer Arbeiten gezeigt, mit dem Konzept der kritischen und isolierenden Kontexte (Diewald 2006a, 2006b).

Bevor dies wieder aufgegriffen wird, sei ein Blick auf das Modell von Konstruktionstypen unterschiedlicher Hierarchiestufen geworfen, das in Traugott (2008a) geboten und in Trousdale (2008a) aufgegriffen wird. Traugott hebt her- 
vor, dass „Konstruktionen [...] voneinander unabhängig, jedoch über ein hierarchisches System mit mehreren Schematizitätsebenen miteinander verbunden [sind]“, so dass sie sich überschneiden können (2008a: 7). Sie schlägt die folgenden vier Hierarchie- bzw. Abstraktionsstufen vor. Dabei sind die ersten drei Stufen auf types, die letzte auf tokens bezogen:

a. Makrokonstruktionen: abstrakte Schemata, die höchststufige Ebene, die für die gegebene Diskussion relevant ist, z.B. die Transitive Konstruktion, die Ditransitive Konstruktion, die Partitive Konstruktion, die Gradmodifizierer-Konstruktion;

b. Mesokonstruktionen: Mengen sich ähnlich verhaltender Konstruktionen, z.B. (a) kind/sort of im Gegensatz zu a bit/ lot of etc.;

c. Mikro-Konstruktionen: Individuelle Konstruktions-Typen, z.B. (a) kind of gegenüber a bit of;

d. Konstrukte: empirisch attestierte Token (,usage based events“ in der Terminologie von Langacker 1987), der Ort von Innovationen. (Traugott 2008a: 8)

Trousdale (2008a) greift dieses Modell auf, indem er die Zahl der „levels of schematic constructions" im Bereich der Meso-Konstruktionen als prinzipiell flexibel bezeichnet und selbst mit zwei „Meso-Ebenen“ arbeitet. Trousdale fasst seine Konzeption wie folgt zusammen:

In this constructional network, constructions display different degrees of schematicity; constructions lower in the taxonomy may inherit properties form those higher in the taxonomy; and constructions intersect (in which case a construction may inherit properties from more than one, supra' construction). The number of constructions in the taxonomy is not (and I would suggest cannot) be fixed, but there is clearly a hierarchy involved. (Trousdale 2008a: 52)

Wie Trousdale (2008a: 44f.) am Beispiel einer „composite predicate construction“ (nämlich der ,give-gerund construction“ wie in I gave him a kicking) im Englischen darstellt, unterscheiden sich die Ebenen grundsätzlich im Ausmaß der lexikalischen Füllung einzelner Positionen: während auf der Ebene der MakroKonstruktionen nur Kategorienbezeichnungen auftreten (z.B. „CP Cxn“ = composite predicate construction oder „DitransitiveCxn“ = ditranstive construction), sind auf den beiden Meso-Ebenen einzelne Slots lexikalisch gefüllt. So ist im Beispiel auf der „meso a“-Ebene nur das Verb, also z.B. „give CPCXn“ oder „give Ditrcxn" spezifiziert, was bedeutet, dass die composite predicate construction bzw. die ditransitive construction in der einschlägigen Position mit dem Verb give besetzt sind. Die „meso b“-Ebene dagegen enthält zusätzlich weitere Spezifikationen, die entweder kategorialer bzw. grammatischer Natur sind, also z.B. die grammatische Form von give spezifizieren (give-gerund Cxn) oder auch weitere lexikalische Fülllungen benennen (z.B. give rise to NP Cxn). Für die Mikro-Ebene schließlich führt Trousdale vollständig spezifizierte Sätze (verstanden als types) an, wie z.B. They gave us a severe dressing down oder She gave me a kiss (beide Seite 45). 
Die hierarchischen Beziehungen zwischen den Ebenen, die als sogenannte „Vererbungsbeziehungen“ gefasst sind, unterscheiden sich also ausschließlich in der Quantität der spezifischen Füllung und in der Anzahl der Konstruktionen, die als „Erblasser“ angesprochen werden müssen. Während die Konstruktionstypen auf der Makroebene wenige bis keine übergeordneten verwandten Konstruktionen aufweisen und nur auf kategoriale Entitäten wie „Satz“ oder „Prädikat“ rekurrieren, beziehen sich die ausformulierten Sätze der Mikroebene auf eine ganze Fülle von verwandten, hierarchiehöheren Konstruktionen. Im Satz She gave me a kiss befänden sich darunter notwendiger Weise auch die Tempus-Konstruktion finiter Verben, die Subjekt-Prädikat-Konstruktion (inklusive eventuell relevanter Kongruenzbeziehungen) etc.

Diese offene netzartige Struktur hat, wie schon erwähnt, zunächst große Vorzüge bei der Modellierung von Sprachwandelvorgängen, die in diesem Modell über eine Veränderung der Hierarchiebeziehungen beschrieben werden können. Dennoch stellt sich die Frage, ob die Ordnung grammatischer Kategorien (also die Existenz grammatischer Paradigmen), denen ja formale, funktionale und kognitive Realität zukommt, durch eine ausschließlich auf diese Art der Hierarchie Bezug nehmende Darstellung nicht zu stark in den Hintergrund gedrängt wird. ${ }^{7}$ Hier wird jedenfalls die Auffassung vertreten, dass auch konstruktionsgrammatische Konzeptionen die essentielle linguistische Information, die in grammatischen Paradigmen enkodiert ist, in geeigneter Weise - zum Beispiel durch Einführung eines weiteren Konstruktionstypus - modellhaft abbilden sollten. Die Notwendigkeit der spezifischen Berücksichtigung grammatischer Paradigmen zeigt sich deutlich in diachronen Grammatikalisierungsstudien. Sie ist der Motor für die Weiterentwicklung des Kontextmodells zu Grammatikalisierungsszenarios, die einen solchen eigenständigen Konstruktionstypus als vierte Stufe vorschlägt.

\section{Kontexte und Konstruktionen bei grammatikalisierendem Sprachwandel}

Mittlerweile ist die Integration konstruktionsgrammatischer Konzepte in Untersuchungen zur Grammatikalisierung durchaus geläufig (vgl. Diewald 2006a, 2006b; siehe auch Literaturangaben in Trousdale 2008a: 33). Diese Entwicklung

$7 \quad$ Diese Frage stellt sich auch für den Ansatz von Rostila 2006, der Grammatikalisierung mit maximaler Schematizität identifiziert und damit die hierarchische Anordnung von Konstruktionen nach ihrer Abstraktheit/Schematizität mit einer Grammatikalisierungsskala gleichsetzt. Vgl. folgendes Zitat: „According to the proposed $\mathrm{CxG}$ view of grammaticalization, all constructions involving schematicity are products of grammaticalization. Furthermore, an implication of this view is that the more schematic a construction is, the more grammaticalized it is. Thus, completely schematic constructions like the ditransitive and transitive constructions in English (cf. Goldberg 1995) should represent the most grammaticalized of all constructions. It will have to be left for future discussion to determine whether such a wide notion of grammaticalization is tenable“ (Rostila 2006: 53). 
ergibt sich folgerichtig aus der schon früh getroffenen Feststellung, dass Sprachwandelphänomene, insbesondere in ihren ersten Phasen, hochgradig kontextsensitiv sind. Um den Prozess der Grammatikalisierung angemessen zu beschreiben, muss also Kontext- bzw. Konstruktionsgebundenheit an zentraler Stelle in das zu entwerfende Grammatikalisierungsszenario eingebunden werden.

Hierzu wurden Modelle entwickelt, die sowohl zum Begriff „Kontext(typ)“ wie auch zur Sukzession von Kontexttypen generalisierbare Aussagen machen. Das von Diewald in mehreren Arbeiten weiterentwickelte Kontextmodell soll im Folgenden kurz dargestellt werden. Es wurde für diachrone Zwecke entwickelt, und projiziert das Konzept kritischer und isolierender Kontexte auf die Differenzierung verschiedener idiomatischer Konstruktionstypen nach Fillmore, Kay und O’Connor (1988). Während das ursprüngliche Modell drei Stufen annahm (Diewald 2002, 2006a, 2006b), wird nun eine vierte Stufe hinzugefügt, so dass folgendes Schema entsteht:

Tabelle (1): Vierstufiges Kontextmodell für Grammatikalisierungsprozesse

\begin{tabular}{|c|c|c|c|}
\hline Stufe & Kontext & Bedeutung/Funktion & $\begin{array}{l}\text { Konstruktionstyp } \\
\text { (nach Fillmore/Kay/ } \\
\text { O'Connor (1988)) }\end{array}$ \\
\hline $\begin{array}{l}\text { I) Vorbedingungen } \\
\text { der Grammatika- } \\
\text { lisierung }\end{array}$ & $\begin{array}{l}\text { untypische } \\
\text { Kontexte }\end{array}$ & $\begin{array}{l}\text { konversationelle } \\
\text { Implikatur }\end{array}$ & $\begin{array}{l}\text { kein eigenständiger } \\
\text { Konstruktionstyp; } \\
\text { kompositional }\end{array}$ \\
\hline $\begin{array}{l}\text { II) Auslösung der } \\
\text { Grammatikalisie- } \\
\text { rung }\end{array}$ & $\begin{array}{l}\text { kritische } \\
\text { Kontexte }\end{array}$ & multiple Opakheit & $\begin{array}{l}\text { "extragrammatical } \\
\text { idioms“" }\end{array}$ \\
\hline $\begin{array}{l}\text { III) Reorganisation } \\
\& \text { Differenzie- } \\
\text { rung }\end{array}$ & $\begin{array}{l}\text { isolierende } \\
\text { Kontexte }\end{array}$ & polysem/heterosem & $\begin{array}{l}\text { „formal or lexically } \\
\text { open idioms“ }\end{array}$ \\
\hline $\begin{array}{l}\text { IV) Paradigmati- } \\
\text { sche Integration }\end{array}$ & $\begin{array}{l}\text { paradigmati- } \\
\text { sche Kon- } \\
\text { texte }\end{array}$ & $\begin{array}{l}\text { paradigmatische Opposi- } \\
\text { tionen mit reduzierter, } \\
\text { relationaler, d.h. gram- } \\
\text { matischer Bedeutung }\end{array}$ & $\begin{array}{l}\text { paradigmatische Wahl } \\
\text { in einer abstrakten } \\
\text { Konstruktion }\end{array}$ \\
\hline
\end{tabular}

Das ursprüngliche Modell umfasste die ersten drei Stufen, wobei Stufe I lediglich Vorbedingungen zu einer möglichen Grammatikalisierung beschreibt, die im Wesentlichen aus einer über konversationellen Implikaturen gesteuerten Ausweitung der Verwendung eines Zeichens/einer Konstruktion bestehen. Während diese Stufe hier nicht weiter beschrieben werden soll, sind einige Erläuterungen zur zweiten und dritten Stufe angebracht, die wie aus der Tabelle ersichtlich, mit verschiedenen idiomatischen Konstruktionen nach Fillmore, Kay \& O’Connor (1988) korreliert sind.

Der kritische Kontext - das ist die zweite Stufe in der relativen Chronologie von Grammatikalisierungsprozessen - bewirkt die Auslösung der Reinterpretation in Richtung auf die neue grammatische Funktion. Es handelt sich um eine 
hochmarkierte Konstruktion, die durch Ambiguität in mehreren Schichten der linguistischen Struktur gekennzeichnet ist, und die verschiedene Interpretationsalternativen zulässt bzw. diese Alternativen geradezu provoziert, wobei die neu entstehende grammatische Bedeutung eine der möglichen Lesartalternativen darstellt.

Kritische Kontexte stellen immer neue und periphere Strukturen dar, die typischerweise auf eine kleine Gruppe lexikalischer Einheiten beschränkt sind und die mit den Mitteln des bisherigen linguistischen Systems nicht vollständig analysierbar sind. Daher kann hier von „extragrammatical idioms“ bzw. extragrammatischen idiomatischen Konstruktionen gesprochen werden (siehe Abschnitt 2). Ein Beispiel für einen kritischen Kontext aus der Entwicklung des Lexems eben zur Modalpartikel ist folgender Satz (nach Diewald \& Ferraresi 2008: 99):

(1) Man befahl, eben die Marter an mir vorzunehmen. (BA Gel 1518, 18th c.)

Es handelt sich um einen hochgradig opaken Kontext mit drei möglichen Lesarten für eben: als Temporaladverb in der Bedeutung ,gerade jetzt', genau zu diesem Zeitpunkt' wie in Paraphrase (2a), als Skalarpartikel, die die nachfolgende NP modifiziert, also ,genau diese Marter' wie in (2b), und als Modalpartikel, d.h. mit einer iterativen Komponente, wie in Paraphrase (2c) expliziert (zu den Modalpartikeln als grammatische Elemente siehe Abschnitt 2):

(2)a. Paraphrase von eben in (1) als Temporaladverb: ,Man befahl, die Marter genau zu diesem Zeitpunkt an mir vorzunehmen‘.

b. Paraphrase von eben in (1) als Skalarpartikel: ,Man befahl, genau diese Marter an mir vorzunehmen‘.

c. Paraphrase von eben in (1) als Modalpartikeln:

„Man befahl, - ich wiederhole Gegebenes - die Marter an mir vorzunehmen“.

Die Zahl der Wahlmöglichkeiten und die Wahrscheinlichkeit für eine bestimmte Interpretation sind variabel; entscheidend für die Annahme eines kritischen Kontexts ist das Vorhandensein von Ambiguität bzw. Opakheit auf mehreren Ebenen, wobei eine von mehrerer Lesartalternativen die neue grammatische Bedeutung zum Ausdruck bringen muss (im obigen Fall die Lesart als Modalpartikel). Der kritische Kontext fungiert als eine Art Katalysator der Grammatikalisierung, der sehr oft in der weiteren Entwicklung wieder verloren geht.

Traugott [demn.] diskutiert das von Diewald entwickelte Konzept des kritischen Kontexts vor dem Hintergrund des Konzepts des „bridging context“ (nach z.B. Heine 2002, Evans \& Wilkins 2000: 549, Eckardt 2006 oder Hansen 2008), der ausschließlich auf semantische Ambiguität gemünzt ist. Die verschiedenen Positionen werden u.a am Beispiel der Entwicklung des englischen going toFuturs überprüft und es zeigt sich, dass die Integration sowohl semantischer wie auch struktureller Ambiguität ein entscheidender Vorteil der Konzeption des kritischen Kontexts ist, da nur so die Entstehung neuer Konstruktionen, die ja auch formale Neuerungen bzw. neue Zuordnungen von Bedeutung und Form enthalten, in ihren sehr spezifischen lokalen Bedingungen (Traugott spricht von „micro- 
steps") erfasst werden kann. Traugotts Untersuchung liefert auch Indizien für die von Diewald postulierte „Kurzlebigkeit“ des kritischen Kontexts als Katalysator (Traugott [demn.]: 15, 21). ${ }^{8}$

Isolierende Kontexte andererseits, die der dritten Stufe von Grammatikalisierungsprozessen zugeordnet sind, sind Kontexte, die eine Lesart stark favorisieren und eine andere Lesart ausschließen. Auf diese Weise wird die neue grammatische Bedeutung als selbständige Bedeutung von der älteren, stärker lexikalischen Bedeutung getrennt, d.h. es entsteht je ein isolierender Kontext für die grammatische Lesart und ein anderer für die lexikalische Lesart.

Die neue, grammatische Bedeutung ist nicht länger von konversationellen Implikaturen abhängig, sondern das in Grammatikalisierung befindliche linguistische Element ist polysem geworden. Diese Phase kennzeichnet die Konsolidierung des Grammatikalisierungsprozesses, d.h. die Reorganisation und Differenzierung der grammatischen Formative und des Paradigmas, das die Zielkategorie dieses laufenden Grammatikalisierungsvorgangs ist. Ein Beispiel hierfür wäre ein Satz wie (3), in dem nur mehr die Lesart von eben als Modalpartikel sinnvoll ist:

(3) Modalpartikeln sind eben so.

Isolierende Kontexte sind formale Idiome (siehe Abschnitt 1), da sie einerseits Teilähnlichkeiten mit anderen Konstruktionen aufweisen, die als Vererbungsbeziehungen aufgefasst werden können, da sie aber andererseits auch eine einmalige Form-Bedeutungs-Korrespondenz aufweisen, die nicht auf die Kombination aus anderen Konstruktionen zurückgeführt werden kann. Isolierende Kontexte sind also teilproduktive, idiomatische Konstruktionen im konstruktionsgrammatischen Sinn. Mit Hilfe dieses Schemas der Kontext- und Konstruktionstypen lassen sich komplexe Grammatikalisierungsvorgänge wie derjenige der Entstehung der Modalpartikeln im Deutschen modellieren.

3. Paradigmatisierung als notwendige vierte Stufe in Grammatikalisierungsprozessen

Die mehrfache Erprobung des dreistufigen Modells hat ergeben, dass es auf zahlreiche Grammatikalisierungsprozesse sinnvoll anwendbar ist. Eine Summe dieser Überprüfung an mehreren Beispielfällen ist in Diewald \& Smirnova [eingereicht a]

8 Als notwendige Modifikationen des Konzepts des kritischen Kontexts nennt Traugott die Möglichkeit, dass diese Stufe in bestimmten Grammatikalisierungsprozessen optional sein könnte und dass es statt eines kritischen Kontexts zugleich mehrere relevante Kontexte in der entscheidenden Phrase geben kann. Hierzu folgendes Zitat: „In sum, „critical“ contexts are more appropriate than „bridging“ contexts in accounting for the onset of grammaticalization. However, a modification of Diewald's hypotheses is called for. The fundamental difference is that Stage II is optional, and critical context is to be understood in an extended sense. It does not imply restriction to one specific context. It should be understood only as pragmatic, semantic, and structural context." (Traugot [demn.: 21]). 
dargelegt, die im Folgenden in tabellarischer Zusammenfassung kurz präsentiert werden. Es handelt sich um zwei Modalpartikeln, die aus Adjektiven bzw. Adverbien entstanden sind (aber und rubig), und um die Entwicklung der Infinitivkonstruktion mit drohen zu einem Evidentialitätsmarker.

Tabelle (2): Beispiele für die Sukzession der Kontexttypen in verschiedenen Grammatikalisierungsprozessen (nach Diewald \& Smirnova [eingereicht a])

(a) aber als MP

\begin{tabular}{|c|c|}
\hline \multicolumn{2}{|l|}{ Ursprung } \\
\hline $\begin{array}{l}\text { 1. der eine [ging] in sin dorf, abir der andere zuo sime gewerbe. } \\
\text { (Evangelienb. Behaim } 51 \text { B. (1343), DWB Neubearb. 1: } \\
\text { 181) }\end{array}$ & $\begin{array}{l}\text { Adverb/Konjunktion mit } \\
\text { temporaler, iterativer und } \\
\text { adversativer Bedeutung }\end{array}$ \\
\hline \multicolumn{2}{|l|}{$\begin{array}{l}\text { Kritischer Kontext (18. Jh.): } \\
\text { exklamative Sprechakte mit V2-Stellung }\end{array}$} \\
\hline $\begin{array}{l}\text { 2. Ibr müßt aber hier jämmerliche Langeweile haben. (GoeWb, } \\
\text { aus Paul/Henne (1992)) }\end{array}$ & $\begin{array}{l}\text { (i) adversative Konjunktion } \\
\text { (ii) kopulative Konjunktion } \\
\text { (iii) Modalpartikel }\end{array}$ \\
\hline \multicolumn{2}{|c|}{$\begin{array}{l}\text { Isolierende Kontexte: } \\
\text { exklamative Sprechakte mit V2- Stellung (a) und V1- Stellung (b), dass-Sätze (c), } \\
\text { Imperativsätze (d) }\end{array}$} \\
\hline $\begin{array}{l}\text { 3. a. Das war aber eine Reise! } \\
\text { b. Schmeckt dieser Wein aber gut! } \\
\text { c. Dass Peter aber (auch) eine solche Reise gemacht hat! } \\
\text { d. Nun schlaf aber (auch) endlich! } \\
\text { (nach Helbig (1994: } 80 \mathrm{ff} .) \text { ) }\end{array}$ & $\begin{array}{l}\text { Modalpartikel mit adver- } \\
\text { sativer Bedeutung }\end{array}$ \\
\hline
\end{tabular}

(b) rubig als MP

\begin{tabular}{|l|l|}
\hline Ursprung \\
\hline 1. Er ist ein rubiger Mensch./Er arbeitet rubig. & Adjektiv/Adverb \\
\hline $\begin{array}{l}\text { Kritischer Kontext (18. Jh.): } \\
\text { morphologischer Imperativ in face-to-face-Dialogen }\end{array}$ \\
\hline $\begin{array}{l}\text { 2. ,Schweig“, rief der Gaul, ,und laß mich rubig pflügen; ... “ } \\
\text { (Das Kutschpferd, Gellert Werke, Bd. 1: 113f.) }\end{array}$ & $\begin{array}{l}\text { (i) Adjektiv } \\
\text { (ii) Adverb } \\
\text { (iii) Modalpartikel }\end{array}$ \\
\hline $\begin{array}{l}\text { Isolierende Kontexte: } \\
\text { Imperativsätze (a), Deklarativsätze mit einem Modalverb (b), hortative Konstruktionen (c) }\end{array}$ \\
$\begin{array}{l}\text { 3. a. Komm rubig herein! } \\
\text { b. Du darfst rubig hereinkommen. (Helbig, s.v. rubig) } \\
\text { c. Sagen wir rubig die Reaktionäre (IDS-DSAV FR200_54) }\end{array}$ \\
\hline
\end{tabular}


(c) drohen als Evidentialitätsmarker

\begin{tabular}{|c|c|}
\hline \multicolumn{2}{|l|}{ Ursprung } \\
\hline $\begin{array}{l}\text { 1. sie tröuwend euch mit dem schwerdt auszureuten und ze } \\
\text { verbrennen (Maaler } 409 c, \mathrm{DWB} \text { ) }\end{array}$ & (Sprechakt-)verb \\
\hline \multicolumn{2}{|l|}{$\begin{array}{l}\text { Kritischer Kontext (18. Jh.): } \\
\text { unbelebte Subjekte und Infinitive mit Handlungssemantik }\end{array}$} \\
\hline $\begin{array}{l}\text { 2. schon loderte die Flamme, und drobte das herrliche Werk in die } \\
\text { Asche } z^{\prime} \text { legen. (Klinger, DE-Gutenberg) }\end{array}$ & $\begin{array}{l}\text { (i) Hauptverb (Metapher) } \\
\text { (ii) temporal-aspektuell } \\
\text { (iii) inferentielle Eviden- } \\
\text { tialität }\end{array}$ \\
\hline \multicolumn{2}{|c|}{$\begin{array}{l}\text { Isolierende Kontexte: } \\
\text { belebte (a) und unbelebte (b) Subjekte der 3.Pers. und Infinitive mit Nicht- } \\
\text { Handlungsverben }\end{array}$} \\
\hline $\begin{array}{l}\text { 3. a. Sitz gerade auf dem Fahrrad, Ija! Führe den Lenker nach } \\
\text { links, wenn du nach rechts ₹u kippen drobst! (IDS-Corpus) } \\
\text { b. aber die Sonnenbitze brennt gar zu stark, daß mir das zarte } \\
\text { Kraut zu welken drobt...(GRI/KHM.00122, IDS-Corpus) }\end{array}$ & $\begin{array}{l}\text { inferentieller Evidentiali- } \\
\text { tätsmarker (Auxiliar) }\end{array}$ \\
\hline
\end{tabular}

Wie ersichtlich ist das Modell der drei Kontext- bzw. Konstruktionsstufen für die unterschiedlichsten grammatischen Elemente relevant. Neben Modalverben, an denen es zunächst erprobt wurde (Diewald 2002), folgen auch andere Verbalkategorien wie z.B. Evidentialitätsmarker (hierzu ausführlich Diewald \& Smirnova [eingereicht b]) und die Klasse der Modalpartikeln (Diewald 2006c, Diewald \& Ferraresi 2008) diesem Szenario.

Im Zuge der Untersuchung hat sich jedoch gezeigt, dass das dreistufige Modell (ohne die vierte Stufe der Paradigmatisierung) insofern zu mächtig ist, als es auch auf bestimmte Typen des semantischen Wandels und der kategorialen Veränderung übertragen werden kann, die nicht als Grammatikalisierungsprozesse zu bezeichnen sind. Beispiele hierfür sind die Entstehung sekundärer Präpositionen aus Syntagmen, z.B. (an)statt aus ,an jemandes Statt“ (Präposition \& NP im Genitiv \& statt), bei denen sich Grammatikalisierung und Lexikalisierung verbinden. ${ }^{9}$

$9 \quad$ Lexikalisierung wird mit Bezug auf Lehmann (2004) verstanden als der Verlust interner Strukturiertheit, d.h. kompositioneller Motivation und Inventarisierung. Lexikalisierung und Grammatikalisierung können in einem Wandelprozess gemeinsam auftreten. Dazu die Ausführungen Lehmanns als Zitat: „Given a construction X-Y Z, in which X-Y is befallen by reduction, then grammaticalization and lexicalization may operate at the same time. Take German aufgrund, on the basis of ${ }^{\prime}$ as an example: $\mathrm{X}=$ auf, $\mathrm{Y}=$ Grund, $\mathrm{Z}=$ the genitive complement of $\mathrm{Y}$. Univerbation of auf + Grund is lexicalization, because it goes against the syntactic structure and destroys it. It transforms the complex into a lexical item of the category ,preposition'. Desemanticization of the result by loss of concrete local features, accompanied by the loss of a couple of nominal properties, is grammaticalization of the fresh lexical item.“" (Lehmann 2004: 169) 
Zum anderen ist auch die Entstehung von Polysemien, wie z.B. beim Adjektiv billig im Deutschen, über das dreistufige Szenario erfassbar, was die folgende Tabelle zeigt:

Tabelle (3): Entwicklung der Polysemie von billig (nach Diewald \& Smirnova [eingereicht a]):

\begin{tabular}{|c|c|}
\hline \multicolumn{2}{|l|}{ Ursprung } \\
\hline $\begin{array}{l}\text { 1. denn er weiß das allein gott dem herrn billich und von recht eere und glo- } \\
\text { by zugehoeret (Kaysersberg, Seelenparadies, } 12 \mathrm{va} \text {, aus Fritz 2005: 114) }\end{array}$ & $\begin{array}{l}\text {,angemessen, rich- } \\
\text { tig, gemäß, passend‘ }\end{array}$ \\
\hline \multicolumn{2}{|l|}{$\begin{array}{l}\text { Kritischer Kontext (17. Jh.): } \\
\text { billig }+ \text {,Preis` in (Zeitungs-)Anzeigen }\end{array}$} \\
\hline $\begin{array}{l}\text { 2. (Alle fremden Händler haben Erlaubnis vom König) ibre Victualien in un- } \\
\text { ser Lager ₹u fübren/ daber alles nun um einen billichen Preis zu bekommen } \\
\text { ist. (Nordischer Mercurius 1667, 382.25, aus Fritz 2005: 115) }\end{array}$ & $\begin{array}{l}\text { (i), angemessen' } \\
\text { (ii) ,preiswert' }\end{array}$ \\
\hline \multicolumn{2}{|l|}{$\begin{array}{l}\text { Isolierende Kontexte (19. Jh.): } \\
\text { billig + ,Preis‘, ,Waren', ,Dienstleistungen` etc. }\end{array}$} \\
\hline 3. Telefonieren kann so billig sein. & ,preiswert ${ }^{6}$ \\
\hline
\end{tabular}

Das heutige Adjektiv billig hat zwei zentrale Bedeutungen, nämlich ,angemessen“ und ,preiswert', wobei die zweite sich aus der ersten, wie in Beispiel (1) in Tabelle 3 ersichtlich, entwickelt hat. Diese Entwicklung fand in einem bestimmten kritischen Kontext statt, der über zwei relevante Merkmale definiert werden kann: (i) das Adjektiv modifiziert das Nomen ,Preis'; und (ii) diese Kombination erscheint vorwiegend in Zeitungsanzeigen (Beispiel 2, Tabelle 3), d.h. in einem bestimmten Textsortenmuster und damit pragmatischen Kontext. In solchen Kontexten war das Adjektiv billig zunächst semantisch ambig zwischen beiden Lesarten. Heute, ist billig, wenn es mit Nomina mit kommerzieller Referenz (Preise, Waren, Dienstleistungen etc.) verbunden ist, ausschließlich auf die zweite, neue Bedeutung beschränkt (Beispiel 3, Tabelle 3), so dass diese Konstruktionen als isolierender Kontexte für die neue Lesart/Bedeutung interpretiert werden können.

Obwohl die Details hier nicht weiter ausgeführt werden können, ist festzuhalten, dass das vorgeschlagene Modell der sukzessiv relevanten Konstruktionstypen nicht nur für Grammatikalisierung, sondern auch für Lexikalisierung gilt und sich darüber hinaus (mit gewissen Vereinfachungen) zur Beschreibung semantischen Wandels eignet. Das heißt, dass mit diesem Modell das distinktive Element von Grammatikalisierungsprozessen noch nicht abschließend erfasst ist.

Das dreistufige Modell ist zwar - wie in den Studien gezeigt werden konnte ein wichtiges deskriptives Instrument für die Rekonstruktion von relevanten Stadien in Grammatikalisierungsprozessen. Insbesondere das Konzept des kritischen Kontextes als semantisch und strukturell ambige Struktur und die dritte Stufe, das Stadium der isolierenden Kontexte, die die neue grammatische Bedeutung als eigenständiges Zeichen verfügbar macht, sind essentiell für Grammatikalisierungsprozesse, da mit diesen Stufen ist die Möglichkeit zur vollständigen Grammatika- 
lisierung, also zur Eingliederung in ein bestehendes oder sich erst konstituierende Paradigma, angezeigt wird.

Jedoch werden im Rahmen des dreistufigen Modells keine Aussagen über den tatsächlichen Vollzug der paradigmatischen Eingliederung, ihre spezifische Ausprägung und die damit verbundene obligatorische Verwendung des neuen Elementes gemacht.

Um die Stufe der Paradigmatisierung, also ein sehr weit fortgeschrittenes (und keineswegs immer erreichtes) Stadium von Grammatikalisierungsprozessen $\mathrm{zu}$ beschreiben, wird, wie schon beschrieben, eine vierte Stufe eingeführt (vgl. Tabelle 1). Diese vierte Stufe erfasst, anders als die vorhergehenden Stufen, nicht mehr den syntagmatischen Kontext, sondern den paradigmatischen Kontext. Sie ist spezifisch für Grammatikalisierung im Gegensatz zu Lexikalisierung und allen anderen Arten von Sprachwandel und erfasst also das Alleinstellungsmerkmal von Grammatikalisierungsprozessen, das in seiner Zielorientierung hin auf einem grammatischen Paradigma beruht.

Diese Stufe wird als das Stadium der paradigmatischen Integration bezeichnet, da hier das neu grammatikalisierte Element in ein (relativ) geschlossenes, (relativ) obligatorisches Paradigma integriert wird. Im Unterschied zu den anderen Phasen, die allesamt die syntagmatische Achse als Kontext nutzen, ist die vierte Stufe auf den paradigmatischen Kontext bezogen. Durch diesen Prozess wird die neue grammatische Bedeutung stabilisiert, insofern als sie, erstens, mit den Oppositionsgliedern des Paradigmas konfrontiert wird, und, zweitens, als sie damit allmählich der abstrakteren, kategorialen Bedeutung unterworfen wird, die den gemeinsamen Nenner des Paradigmas (und aller seiner Mitglieder) bildet. Die daraus resultierende Struktur ist dann exakt das fest integrierte Paradigma, das Lehmann beschreibt:

On the semantic side, the members of a paradigm have a common semantic basis with varying differentiae specificae. This would be brought out by a componential analysis and is reflected in traditional terminology by the fact that there is a generic category name for the whole paradigm and opposite names for the specific subcategories. Such paradigmacity is gradually reached in the process of grammaticalization. Categories grammaticalized very little do not constitute such tightly integrated paradigms. (Lehmann 2002: 120)

Anders formuliert: auf der vierten Stufe wird die neue grammatische Bedeutung der abstrakten Bedeutung des Gesamtparadigmas unterworfen und verliert somit ihre Autonomie. Innerhalb des Paradigmas nimmt sie nun eine spezifische Position ein, die sie in Opposition zu den übrigen Mitgliedern des Paradigmas setzt. Die Bedeutung der neuen Form ist determiniert von diesen beiden Faktoren (dem Hypernym und den Oppositionsgliedern). Die neue Bedeutung wird also in das Paradigma integriert, indem sie paradigmatische Beziehungen zu den anderen Mitgliedern des Paradigmas aufnimmt, wodurch das Paradigma als Ganzes verändert wird. 
Dieser Sachverhalt kontrastiert scharf mit Vorgängen bei Lexikalisierungsprozessen, da bei letzteren (also der Inventarisierung einer Einheit im Lexikon), ausschließlich semantischer Wandel vorliegt (wie z.B. oben anhand von billig illustriert), ohne dass eine abstrakte Bedeutung in vergleichbarer Weise als integrierender Oberbegriff des Paradigmas steuernd wäre und ohne dass das Gesamtgefüge maßgeblich modifiziert würde. Kurz: Bei Lexikalisierung ist kein Paradigma im eigentlichen Sinne vorhanden.

Wenn die neu entstehende Form in ein bereits bestehendes grammatisches Paradigma integriert wird, wenn also bereits eine Zielkategorie existiert, liegt nach Lehmann (1995[1982]: 21) „renovation“ oder „renewal“ vor. Dies ist der Fall bei der Entwicklung der periphrastischen Tempora im Deutschen und bei der Entwicklung der grammatikalisierten Verwendung der Modalverben.

Existiert dagegen keine Zielkategorie und kein entsprechendes grammatisches Paradigma, dann entsteht durch den Grammatikalisierungsprozess eine völlig neue grammatische Kategorie in der betreffenden Sprache, wobei die älteren Mitglieder des neuen Paradigmas im Allgemeinen das Modell für die jüngeren abgeben, dem sich diese oft auch durch Beteiligung analogischen Wandels anschließen. Dieser Prozess einer Neuschöpfung wird mit Lehmann „Innovation“ genannt (Lehmann 1995[1982]: 21).

Die Existenz eines bereits bestehenden Paradigmas der Zielkategorie kann im Gange befindliche Grammatikalisierungsprozesse beschleunigen. Dies wurde z.B. in Diewald (1999) für die Integration der Modalverben ins Modusparadigma gezeigt. Für die Modalverben, deren isolierende Kontexte bei allen betroffenen Verben spätestens im 17. Jahrhundert vorlagen, besteht diese vierte Stufe vor allem in der Ausdifferenzierung der jeweiligen modalen Werte in Opposition zu den anderen grammatikalisierten Modalverbverwendungen (also z.B. der Herausbildung der spezifischen formalen und funktionalen Restriktionen bei dürfe und mag, der Entwicklung der spezifischen quotativen bzw. reportiven Funktionen von soll und will etc.). Diese ist in Tabelle 4 wiedergegeben.

Tabelle (4): Die paradigmatische Organisation der Modusmarkierungen im Deutschen

\begin{tabular}{|l|l|c|c|c|c|}
\hline \multicolumn{2}{|c|}{ Kategorie Modus } & \multicolumn{2}{|c|}{$\begin{array}{c}\text { semantische Oppositionen im Paradigma } \\
\text { (distinktive Werte der einzelnen Mitglieder) }\end{array}$} \\
\cline { 3 - 6 } & faktisch & \multicolumn{3}{|c|}{ nicht-faktisch } \\
\cline { 3 - 6 } & $\begin{array}{l}\text { flexivisch: } \\
\text { innere und } \\
\text { außere } \\
\text { formale } \\
\text { Realisierung } \\
\text { Flexion }\end{array}$ & Indikativ & phorisch & quotativ \\
\hline
\end{tabular}




\begin{tabular}{|l|l|l|c|c|c|}
\hline \multirow{2}{*}{$\begin{array}{l}\text { (verbale } \\
\text { Modus- } \\
\text { markierung) }\end{array}$} & $\begin{array}{l}\text { periphrastisch: } \\
\text { würde \& } \\
\text { Infinitiv }\end{array}$ & & & $\begin{array}{c}\text { würde \& } \\
\text { Infinitive }\end{array}$ & \\
\cline { 2 - 5 } & $\begin{array}{l}\text { periphrastisch: } \\
\text { Modalverb \& }\end{array}$ & & können \\
& müssen & $\begin{array}{c}\text { dürfte } \\
\text { mag }\end{array}$ & $\begin{array}{c}\text { sollen } \\
\text { wollen }\end{array}$ \\
\hline
\end{tabular}

Unabhängig davon, wie die Werte und Oppositionen der einzelnen Mitglieder zu interpretieren sind (der Vorschlag in der Tabelle folgt den Ergebnissen der Untersuchung von Diewald (1999), die u.U. in Teilen zu modifizieren sind), zeigt dieses Beispiel das Prinzip der paradigmatischen Integration und die Wichtigkeit von Stufe IV im Grammatikalisierungsmodell. Was den historischen Ablauf dieses Prozesses betrifft, so konnte Diewald (1999) zeigen, dass die paradigmatische Eingliederung in enger gegenseitiger Beeinflussung (durch analogischen Ausgleich) und unter den Restriktionen der bereits paradigmatisch organisierten Zielkategorie (des verbalen Modus) statt fand. Als formender Zeitraum können das 18. und 19. Jahrhundert angenommen werden, wobei selbstverständlich auch in späteren Epochen weitere Entwicklungen stattfinden (können). Hier sind jedoch weitere umfangreiche Korpusanalysen erforderlich.

Während die Modalverben in ein bestehendes Paradigma integriert wurden und somit den Fall der Renovation illustrieren, zeigt die Entstehung des Paradigmas der evidentiellen Konstruktionen im Deutschen den Fall der Innovation, also die Entstehung eines neuen Paradigmas. Dies ist im Übrigen auch bei der Entstehung des Passivs, der Modalpartikeln und des Artikels der Fall. Im Falle der paradigmatischen Integration einer Einheit, die zugleich in einer anderen, stärker lexikalischen Form weiter existiert (also „Persistenz“ aufweist), liegt Heterosemie, also die Spaltung in verschiedene funktionale Klassen vor. Dies ist bei etlichen Modalpartikeln der Fall und kann auch für die parallele Existenz von Vollverbund Auxiliardoubletten z.B. bei werden und anderen Hilfsverben angenommen werden.

Anzumerken ist schließlich, dass die Stufe der paradigmatischen Integration keine scharf abgegrenzte zeitliche Epoche ist, sondern eine kontinuierliche Entwicklung über einen längeren Zeitraum darstellt. Zu Beginn ist mit formal inhomogenen Paradigmen zu rechnen, die zahlreiche Mitglieder und relativ reichhaltige, überlappende Bedeutungen aufweisen; ältere Paradigmen hingegen zeichnen sich durch größere formale Homogenität, geringere Mitgliederzahl und abstrakte Bedeutungsstrukturen aus, die in klaren Oppositionen bzw. Markiertheitshierarchien stehen. 
4. Das grammatische Paradigma als Konstruktion

Die im letzten Abschnitt beschriebene enge semantische Organisation eines grammatischen Paradigmas ist ohne Zweifel seine markanteste und funktional wichtigste Eigenschaft. Was die formalen Eigenschaften von Mitgliedern eines Paradigmas betrifft, so können sie - wie illustriert - sehr unterschiedlicher Natur sein und zum Beispiel flexivische und periphrastische (analytische) Formen mischen. Die Paradigmen der deutschen Tempus- und Moduskategorien sind hier ein eindrucksvolles Beispiel (vgl. Tabelle 4 für Modus): beide enthalten Elemente, die sich durch „innere Flexion“ (Wechsel des Stammvokals durch Ablaut oder Umlaut) auszeichnen, ebenso wie solche, die Affigierung aufweisen. Hinzu kommen drittens auch zahlreiche analytische Formen.

Die erste formale Option - innere Flexion - ist realisiert in Präteritalformen wie schwamm, fubr oder Modusformen wie gäbe oder fübre, die flexivische Option ist realisiert in den Tempusformen des schwachen Präteritums wie z.B. lachte, fragte, oder in Modusformen wie er lache, er frage. Die analytische Option schließlich ist realisiert in Perfektformen (er hat gelacht) und der sogenannten würde-Umschreibung (er würde lachen). Man kann zwar davon ausgehen, dass, je älter ein grammatisches $\mathrm{Pa}$ radigma, desto stärker die formale Homogenität seiner Mitglieder ausgeprägt ist, doch verdeutlicht schon allein dieser kurze Blick auf das formale Realisierungsspektrum, dass die semantisch-funktionale Komponente, die hier als Paradigmatisierung beschrieben ist, das wesentliche Merkmal ist und die formale Ausprägung eines Paradigmas nachgeordnetes Gewicht hat.

Daher wird der Konstruktionstyp der vierten Stufe der Grammatikalisierung, also der Stufe der paradigmatischen Auswahl (d.h. der Existenz eines durch Optionen und Oppositionen geprägten Paradigmas), als ein abstraktes konstruktionales Schema im Sinne Langackers $(1978,1991,2008)$ beschrieben. Dieses abstrakte Schema bezieht sich zugleich auf das Paradigma als Ganzes und auf jedes einzelne seiner Mitglieder.

Ein konstruktionales Schema hat als gemeinsame semantische Basis die kategoriale Bedeutung des Paradigmas (z.B. „Tempusmarkierung“) und eine sehr abstrakte Bestimmung seiner formalen bzw. strukturellen Eigenschaften (z.B. verbale Flexion oder Periphrase). Damit ist der Typus der nicht-reduzierbaren Verbindung von Form und Bedeutung hergestellt, der das entscheidende Merkmal für eine Konstruktion ist. Die Notierungskonvention, die hier als erster Versuch vorgeschlagen wird, enthält folgende Bestimmungen:

- $\quad$ eine Kategorienbezeichnung bzw. Paradigmenbezeichnung, z.B. Tempus

- eine Benennung der abstrakten formalen Eigenschaften der Mitglieder der Kategorie/des Paradigmas

- eine Benennung der gemeinsamen semantisch-funktionalen Merkmale des gesamten Paradigmas. 
Am Beispiel der Tempuskategorie des Deutschen kann dieses Schema wie folgt angewandt werden:

KATEGORIENBEZEICHNUNG: Tempus

FORMALE EIGENSCHAFTEN: modifiziert das Hauptverb durch äußere oder innere Flexion (z.B. Affix -te oder Ablaut wie in schreibe - scbrieb) oder durch periphrastische Formen (baben/ sein + Partizip II etc.)

KATEGORIENBEDEUTUNG: temporale Einordnung des dargestellten Sachverhalts in Relation zur Sprechzeit

Auf dieser Grundlage kann dann jedem Mitglied des Paradigmas eine spezifische formale und semantische Ausprägung zugewiesen werden, die je nach Bedarf auch Besonderheiten und Irregularitäten erfassen kann.

Die vierte Stufe - die paradigmatische Integration - bezieht sich somit auf eine paradigmatisch verknüpfte Gruppe von Einzelkonstruktionen, d.h. auf das Netzwerk an Oppositionsgliedern, die das Paradigma bilden, einerseits und auf jedes einzelne Mitglied/jede einzelne Konstruktion andererseits.

Wichtig hierbei ist, dass die Konzeptualisierung der paradigmatischen Oppositionen, die die grammatische Kategorie ausmachen, als spezifischer Bestandteil der Form-Bedeutungs-Zuordnung bereits in der Konstruktion angelegt sind, d.h. es handelt sich hier um eine spezifische Art der Konstruktion, die eben die paradigmatische Auffächerung enthält. Diese Information ist durch die Einführung der Kategorienbezeichnung in das Schema integriert, d.h. die Zuweisung eines grammatischen Kategorienlabels, wie z.B. Tempus enthält die Information, dass es sich um paradigmatisch organisierte, in der Mitgliederzahl geschlossene, meist numerisch exakt festgelegte, abstrakte grammatische Oppositionen handelt. Diese Analyse muss natürlich unabhängig von der Untersuchung einzelner Paradigmen stattfinden, d.h. auch die Konzeption der letzten Stufe von Grammatikalisierungsprozessen als spezifischer Konstruktionstyp löst nicht das Grundproblem, wie eine grammatische Kategorie prinzipiell zu definieren und „ontologisch“ zu begründen sei. ${ }^{10}$ Dieses Problem ist jedoch nicht das Thema dieses Beitrags, so dass im Folgenden die beispielhafte Anwendung der konstruktionellen Beschreibung auf das oben schon dargestellte Modusparadigma erfolgen kann. Die genannten Bestimmungen werden in der ersten Zeile in die erste Spalte als übergeordnete Konstruktionsmerkmale eingetragen:

10 Die Frage nach dem zugrundeliegenden Grammatikbegriff und damit verbunden der Definition einer grammatischen Kategorie wird in der Literatur zur Grammatikalisierung erstaunlich selten diskutiert. Bereits Himmelmann (1992: 2) äußerst sich hierzu kritisch und stellt fest: „Work in grammaticalization also hardly ever makes explicit the concept of grammar underlying a given investigation." Vgl. hierzu Diewald 2008a und Diewald [im Druck], wo das Problem ausführlich und mit dem Anspruch, einen für Grammatikalisierungsprozesse geeigneten Grammatikbegriff zu erarbeiten, behandelt wird. 
Tabelle (5): Die paradigmatische Organisation der Modusmarkierungen im Deutschen als Konstruktion

\begin{tabular}{|c|c|c|c|c|c|}
\hline \multirow{3}{*}{\multicolumn{2}{|c|}{$\begin{array}{l}\text { KATEGORIENBEZEICHNUNG: } \\
\text { Modus } \\
\text { FORM: modifiziert das Hauptverb } \\
\text { BEDEUTUNG: sprecherbasierte } \\
\text { Faktizitätsbewertung der } \\
\text { Proposition }\end{array}$}} & \multicolumn{4}{|c|}{$\begin{array}{l}\text { semantische Oppositionen im Paradigma } \\
\text { (distinktive Werte der einzelnen Mitglieder) }\end{array}$} \\
\hline & & \multirow[t]{2}{*}{ faktisch } & \multicolumn{3}{|c|}{ nicht-faktisch } \\
\hline & & & $\begin{array}{l}\text { rein } \\
\text { deiktisch }\end{array}$ & phorisch & quotativ \\
\hline \multirow{3}{*}{$\begin{array}{l}\text { formale } \\
\text { Realisierung } \\
\text { (verbale Modus- } \\
\text { markierung) }\end{array}$} & $\begin{array}{l}\text { flexivisch: } \\
\text { innere und } \\
\text { äußere } \\
\text { Flexion }\end{array}$ & Indikativ & & $\begin{array}{l}\text { Konjunktiv } \\
\text { II }\end{array}$ & $\begin{array}{l}\text { Konjunktiv } \\
\text { I }\end{array}$ \\
\hline & $\begin{array}{l}\text { periphrastisch: } \\
\text { würde \& } \\
\text { Infinitiv }\end{array}$ & & & $\begin{array}{l}\text { würde \& } \\
\text { Infinitiv }\end{array}$ & \\
\hline & $\begin{array}{l}\text { periphrastisch: } \\
\text { Modalverb \& } \\
\text { Infinitiv }\end{array}$ & & $\begin{array}{l}\text { können } \\
\text { müssen }\end{array}$ & $\begin{array}{c}\text { dürfte } \\
\text { mag }\end{array}$ & $\begin{array}{l}\text { sollen } \\
\text { wollen }\end{array}$ \\
\hline
\end{tabular}

Dieses Schema kann auf alle grammatischen Kategorien angewendet werden. Die konkrete Realisierung der Tabelle, das heißt die Zahl der Differenzierungen und Spalten ist je nach Kategorie festgelegt und gibt Auskunft über die Zahl der paradigmatischen Oppositionen (als weiteres Beispiel wird in Diewald \& Smirnova [eingereicht a] das Paradigma der inferentiellen Evidentialitätsmarker im Deutschen (also Formen wie Sie scheint/droht/verspricht abgereist zu sein) dargestellt, was hier aus Platzgründen unterbleibt.

Die paradigmatische Konstruktion einer grammatischen Kategorie weist in etwa den Abstraktionsgrad auf, den Traugott für Makrokonstruktionen angibt (siehe Abschnitt 1), so dass man das Konzept der paradigmatischen Konstruktion als Makrokonstruktion betrachten könnte und die Kategorienmitglieder (also z.B. Präsens, Präteritum, Perfekt) als Mesokonstruktionen erster Stufe. Die jeweiligen lexikalisch determinierten semantischen und formalen Restriktionen (z.B. die Verteilung des haben- und sein-Perfekts) würden dann mit zunehmender Spezifizierung niedrigere Positionen in der Hierarchie einnehmen, um schließlich bei den Realisierungsformen der jeweiligen Kategorie pro lexikalische Einheit (z.B. Modusform für den Konjunktiv II beim Verb backen) als Mikrokonstruktion zu enden. Im Gegensatz zum Modell von Traugott und seiner Adaptation durch Trousdale, das in Abschnitt 1 beschrieben wurde, sind paradigmatische Konstruk- 
tionen jedoch insofern enger bestimmt, als sie die Zahl der Füllungsmöglichkeiten an der entscheidenden Stelle definitiv festlegen. Das heißt, die Bedeutung und Funktion der Kategorie und damit der Konstruktion sind durch diese Beschränkung, die auf einen numerischen, endlichen Wert festgelegt wird, definiert. Diese Tatsache und der Umfang der Auswahl ist Bestandteil der Konstruktionsbedeutung. Die Bedeutung der paradigmatischen Konstruktion ist wesentlich durch die Oppositionen und auch die Zahl ihrer Mitglieder definiert, d.h. durch die Kontraste innerhalb ihrer untergeordneten Konstruktionen. Bei den nicht-paradigmatischen Makrokonstruktionen verhält es sich anders: hier ist jede syntagmatische Position im Prinzip mit einer unbeschränkten Zahl von Elementen zu füllen, die nur wenigen Restriktionen unterworfen sind. Die Makrokonstruktion ist nicht paradigmatisch durch die Oppositionen ihrer festgelegten Mitglieder definiert.

Eine weitere Eigenschaft paradigmatischer Konstruktionen, die ein zusätzliches Argument dafür ist, diese als eigenen Konstruktionstyp einzuführen und von der in Abschnitt 1 beschriebenen Konzeption abzugrenzen, betrifft eine spezifische inhärente Eigenschaft grammatischer Einheiten, die hier nur angedeutet werden kann, nämlich, die notwendige Existenz einer relationalen Komponente, also die Tatsache, das grammatische Elemente immer auf andere Einheiten, die sie „modifizieren“, bezogen sind. Dieser Punkt, der in Diewald [im Druck] ausführlich diskutiert wird, ist ohne Zweifel ein Faktor, der die Spezifik grammatischer Paradigmen und damit auch entsprechender Konstruktionstypen determiniert. Seine Einbeziehung in ein Modell für grammatische Konstruktion ist ein Desiderat für zukünftige Arbeiten.

\section{Literatur}

Bergs, Alexander \& Gabriele Diewald (eds.). 2008. Constructions and Language Change. [Trends in Linguistics. Studies and Monographs 194]. Berlin/New York: Mouton de Gruyter.

Bisang, Walter. 1998. „Grammaticalization and language contact, constructions and positions“. In: Anna Giacalone Ramat and Paul Hopper (eds.). The Limits of Grammaticalization. Amsterdam/Philadelphia: Benjamins. 13-58.

Bisang, Walter, Nikolaus P. Himmelmann \& Björn Wiemer (eds.). 2004. What makes Grammaticalization? A Look from its Fringes and its Components. Berlin/New York: Mouton de Gruyter.

Bybee, Joan L., Revere D. Perkins \& William Pagliuca. 1994. The Evolution of Grammar. Tense, Aspect and Modality in the Languages of the World. Chicago: Univ. of Chicago Press.

Croft, William. 2000. Explaining Language Change. An Evolutionary Approach. Harlow etc.: Longman.

Croft, William. 2001. Radical construction grammar. Syntactic theory in typological perspective. Oxford: Oxford University Press. 
Croft, William. 2005. „Logical and typological arguments for Radical Construction Grammar". In: Jan-Ola Östman \& Mirjam Fried (eds.). Construction Grammars. Cognitive Grounding and Theoretical Extensions. Amsterdam/Philadelphia: Benjamins. 273-314.

Dahl, Östen. 2000. The tense-aspect systems of European languages in a typological perspective. In: Östen Dahl (ed.). Tense and Aspect in the Languages of Europe. Berlin/New York: Mouton de Gruyter. 3-25.

Dahl, Östen. 2001. Grammaticalization and the life cycles of constructions. RASK 14. 91133.

Diewald, Gabriele. 1999. Die Modalverben im Deutschen. Grammatikalisierung und Polyfunktionalität. Tübingen: Niemeyer.

Diewald, Gabriele. 2002. „A model for relevant types of contexts in grammaticalization“. In: Ilse Wischer \& Gabriele Diewald (eds.). VERLAG ORT. 103-120.

Diewald, Gabriele. 2006a. „Konstruktionen in der diachronen Sprachwissenschaft.“ In: Kerstin Fischer and Anatol Stefanowitsch (eds.). Konstruktionen. Tübingen: Stauffenburg. 79-103.

Diewald, Gabriele. 2006b. Context types in grammaticalization as constructions. In: Doris Schönefeld (ed.).Constructions. Special Volume 1. Constructions all over-case studies and theoretical implications. [http://www.constructions-online.de/articles/specvol1/].

Diewald, Gabriele. 2006c. „Discourse particles and modal particles as grammatical elements“. In: Kerstin Fischer (ed.). Approaches to discourse particles. Amsterdam [u.a.]: Elsevier. 403-425.

Diewald, Gabriele. 2008a. „Grammatikalisierung, Grammatik und grammatische Kategorien. Überlegungen zur Entwicklung eines grammatikalisierungsaffinen Grammatikbegriffs“. In: Thomas Stolz (ed.). Grammatikalisierung und grammatische Kategorien. Brockmeyer: Bochum (Diversitas Linguarum 21). 1-32.

Diewald, Gabriele. 2008b. „The catalytic function of constructional restrictions in grammaticalization“. In: Elisabeth Verhoeven, Stavros Skopeteas, Yong-Min Shin, Yoko Nishina, and Johannes Helmbrecht (eds.). Studies on grammaticalization. Berlin: de Gruyter. (Trends in Linguistics, Studies and Monographs 2005). 219-240.

Diewald, Gabriele [im Druck]. „On some problem areas in grammaticalization theory“. In: Ekkehard König, Elke Gehweiler \& Katerina Stathi (eds.). What's New in Grammaticalization. Amsterdam: Benjamins. (Studies in Language Companion Series).

Diewald, Gabriele \& Gisella Ferraresi. 2008. „Semantic, syntactic and constructional restrictions in the diachronic rise of modal particles in German: A corpus-based study on the formation of a grammaticalization channel“. In: Seoane, Elena \& María José López-Couso (eds.). Theoretical and Empirical Issues in Grammaticalization. Typological Studies in Language 77. Amsterdam/Philadelphia: Benjamins. 77-110.

Diewald, Gabriele \& Elena Smirnova [eingereicht a]. „Paradigmatic integration: the fourth stage in an expanded grammaticalization scenario“. In: Bert Cornillie et al. (eds.). New Reflections on Grammaticalization IV. Amsterdam/Philadelphia: Benjamins (TSL).

Diewald, Gabriele \& Elena Smirnova [eingereicht b]. Evidentiality in German. Linguistic Realization and Regularities in Grammaticalization. Berlin/New York: Mouton de Gruyter (Trends in Linguistics, Studies and Monographs).

Eckardt, Regine. 2006. Meaning Change in Grammaticalization: An Enquiry into Semantic Reanalysis (Oxford Linguistics). Oxford/New York: Oxford University Press.

Evans, Nicholas \& David Wilkins. 2000. In the mind's ear. The semantic extensions of perception verbs in Australian languages. Language 76. 546-592. 
Fillmore, Charles J., Paul Kay \& Mary C. O’Connor. (1988). „Regularity and idiomaticity in grammatical constructions. The case of let alone“. Language 64/3. 501-538.

Fried, Mirjam \& Jan-Ola Östman. 2004. „Construction Grammar. A thumbnail sketch“. In: Mirjam Fried \& Jan-Ola Östman (eds.). Construction Grammar in a CrossLanguage Perspective. Amsterdam/Philadelphia: Benjamins (CAL 2). 11-86.

Goldberg, Adele. 1995. A Construction Grammar Approach to Argument Structure. Chicago: Chicago University Press.

Goldberg, Adele. 2006. Constructions at Work. The Nature of Generalization in Language. Oxford: Oxford University Press.

Hansen, Maj-Britt Mosegaard. 2008. Particles at the Semantics/Pragmatics Interface: Synchronic and Diachronic Issues: A Study with Special Reference to the French Phasal Adverbs. (Current Research in the Semantics/Pragmatics Interface, 19.) Oxford: Elsevier.

Haspelmath, Martin. 1998. „Does grammaticalization need reanalysis?“ Studies in Language 22. 315-351.

Heine, Bernd. 2002. On the role of context in grammaticalization. In: Ilse Wischer \& Gabriele Diewald (eds.). New Reflections on Grammaticalization. International Symposium. Potsdam, 17-19 June, 1999. Amsterdam/Philadelphia: Benjamins (TSL 49). 83101.

Heine, Bernd. 2003. „Grammaticalization.“ In: Brian Joseph \& Richard D. Janda (eds.). The Handbook of Historical Linguistics. Oxford: Blackwell. 575-601.

Himmelmann, Nikolaus. 1992. Grammaticalization and Grammar. Arbeitspapiere 16 (Neue Folge). Institut für Sprachwissenschaft Universität zu Köln.

Himmelmann, Nikolaus. 2004. „Lexicalization and grammaticalization. Opposite or orthogonal?“. In: Walter Bisang, Nikolaus Himmelmann, Björn Wiemer (eds.). What makes Grammaticalization? A Look from its Fringes and its Components. Berlin/New York: Mouton de Gruyter. 21-42.

Joseph, Brian \& Richard D. Janda (eds.). 2003. The Handbook of Historical Linguistics. Oxford: Blackwell.

Kay, Paul \& Charles J. Fillmore. 1999. „Grammatical constructions and linguistic generalizations. The What's X doing Y? construction“. In: Language 75. 1-33.

Lehmann, Christian. 1985. "Grammaticalization: Synchronic Variation and Diachronic Change". In: Lingua e Stile 20. 303-318.

Lehmann, Christian. 1995 [1982]. Thoughts on grammaticalization. Revised and expanded version. First published edition. München [u.a]: Lincom Europa.

Lehmann, Christian. 1992. "Word order change and grammaticalization.” In: Marinel Gerritsen \& Dieter Stein (eds.). Internal and External Factors in Syntactic Change. Berlin/New York: Mouton de Gruyter, 395-416.

Lehmann, Christian. 2002. Thoughts on grammaticalization. Second, revised edition. Erfurt: Seminar für Sprachwissenschaft der Universität (ASSidUE, 9).

Lehmann, Christian. 2004. „Theory and method in grammaticalization.“ In: Gabriele Diewald (ed.). Grammatikalisierung (Themenheft der Zeitschrift für germanistische Linguistik = ZGL 32). Berlin/New York: de Gruyter. 152-187.

Mel'čuk, Igor A. 1976. „On suppletion“. In: Linguistics 170, 45-90.

Michaelis, Laura. 2004. „Type shifting in construction grammar: An integrated approach to aspectual coercion." In: Cognitive Linguistics 15. 1-67.

Plungian, Vladimir A. 1998. Грамматические категории, их аналоги и заместители. Диссертация на соискание ученой степени доктора филологических наук. Москва, 
1998. [„Grammatische Kategorien, ihre Analoge und Alternativen“. Habilitationsschrift. Moskau 1998].

Radtke, Petra. 1998. Die Kategorien des deutschen Verbs. Zur Semantik grammatischer Kategorien. Tübingen: Narr.

Rostila, Jouni. 2006. Storage as a way to grammaticalization. In: Constructions 1/2006 (www.constructions-online.de).

Traugott, Elizabeth Closs. 2003. „Constructions in Grammaticalization“. In: Joseph, Brian \& Richard D. Janda (eds.). The Handbook of Historical Linguistics. Oxford: Blackwell. 624-647.

Traugott, Elizsabeth Closs. 2008a. „The grammaticalization of NP of NP Patterns“. In: Alexander Bergs \& Gabriele Diewald (eds.). Constructions and Language Change. Berlin: de Gruyter. 23-45.

Traugott, Elizabeth Closs. 2008b. Grammatikalisierung, emergente Konstruktionen und der Begriff der „Neuheit“. In: Kerstin Fischer \& Anatol Stefanowitsch (eds.). Konstruktionsgrammatik II: Von der Konstruktion zur Grammatik. Tübingen: Stauffenburg. 5-32.

Traugott, Elizabeth Closs [demn.]. The status of onset contexts in analysis of microchanges [Manuscript, Draft version, August $8^{\text {th }}$ 2008].

Trousdale, Graeme. 2008a. Constructions in grammaticalization and lexicalization. evidence from the history of a composite predicate in English. In: Graeme Trousdale, Graeme \& Nikolas Gisborne (eds.). 2008. Constructional approaches to English grammar. Berlin/New York: Mouton de Gruyter. 33-67.

Trousdale, Graeme \& Nikolas Gisborne (eds.). 2008. Constructional approaches to English grammar. Berlin/New York: Mouton de Gruyter.

Wiemer, Björn \& Walter Bisang. 2004. „What makes grammaticalization? An appraisal of its components and its fringes?"“. In: Walter Bisang, Nikolaus Himmelmann \& Björn Wiemer (eds.). 2004. What makes Grammaticalization? A Look from its Fringes and its Components. Berlin/New York: Mouton de Gruyter. 3-20.

Wischer, Ilse \& Gabriele Diewald (eds.). 2002. New Reflections on Grammaticalization. International Symposium, Potsdam, 17-19 June, 1999. Amsterdam/Philadelphia: Benjamins (TSL 49).

Adresse der Verfasserin:

Prof. Dr. Gabriele Diewald, Deutsches Seminar, Germanistisches und Angewandte Linguistik, Leibniz Universität Hannover, Königsworther Platz 1, D-30167 Hannover.

E-Mail:gabriele.diewald@germanistik.uni-hannover.de 